\title{
Left Ventricular Diastolic Dysfunction In Hypertension, Diabetes Mellitus And Ischaemic Heart Disease
}

\author{
${ }^{*}$ R.K. Shah, A. B. Upadhyaya, L. P. Tibrewala, P.R. Regmi, K. P. Aeharya, \\ H.H. Khanal, S Rajbhandari, B. Nepal

\section{Background}

Congestive heart failure caused by a predominant abnormality in diastolic function is both common and causes significant morbidity and mortality. However, there is a continued controversy surroundings the definition of diastolic dysfunction and the diagnostic criteria for diastolic heart failure. Recent clinical studies have provided sufficient data to develop standardized diagnostic criteria to define diastolic heart failure.

\section{Materials and Methods}

A prospective analysis was done in 160 consecutive patients who attended Medicare National Hospital And Research Center and NAMS, Bir Hospital, 55 male and 45 female with hypertension (HTN) or diabetes Mellitus (DM), or Ischaemic heart disease (IHD) included in the study while patients having valvular heart disease and cardiomyopathy were excluded from this study. 36 male and 24 female, not having HTN, DM or IHD were taken as control group.

\section{Result}

Male and Female Ratio in Study group was 1:1.2 while in control, group was 1:1.5. Mean age of study group was 58.4 years and 56.8 years in control group, Age ranges from 32 to 78 years. Diastolic dysfunction found in $27(77 \%)$ of Diabetic group ( $n=35), 32$ (71 $\%)$ in Hypertensive group $(n=45)$ and $11(55 \%)$ in Ischaemic group $(n=20)$ and $3(5 \%)$ in control group $(n=60)$. 
- NEPALESE HEART JOURNAL

\section{Conclusion}

1. Left ventricular diastolic dysfunction was most commonly seen in diabetic patients and least commonly in ischaemic group.

2. More than $2 / 3$ of hypertensive patients had diastolic dysfunction.

3. Presence of diastolic dysfunction in general population is low (5\%).

National Academy of Medical Sciences, Bir Hospital, Nepal,

Medicare National Hospital \& Research Centre, Nepal 